3 F. C. G. Sudarshan, Phys. Rev. 123, 2183 [1961]. Eine Diskussion älterer Arbeiten über indefinite Metrik wird in dem Buch gegeben: K. L. NAGY : State Vector Spaces with Indefinite Metric in Quantum Field Theory. Académiai Kiadó, Budapest 1965.

${ }^{4}$ H. P. Dürr u. E. Rudolph, Nuovo Cim. 62, 411 [1969]; 65, 423 [1970]; E. Rudolph, Diplomarbeit München 1967.

5 C. N. Yang u. R. L. Mills, Phys. Rev. 96, 191 [1954]; R. Utiyama, Phys. Rev. 101, 1597 [1956]; E. Rudolph, Doktorarbeit München 1970; E. RUdolph u. H. P. DÜRR, Gupta-Bleuler Formalism with Scalar Fermions in Massless Yang-Mills Theory, Nuovo Cim. 10 A, 597 [1972].

6 R. P. Feynman, Acta Phys. Polon. 24, 697 [1963]; B. S. DE WITT, Phys. Rev. 162, 1195, 1239 [1967]; 171, 1834 E [1968] ; L. D. Fadeev u. V. N. Popov, Phys. Lett. 25 B, 29 [1967].

7 S. Mandelstam, Ann. Phys. 19, 1, 225 [1962] ; Phys. Rev. $175,1580,1604$ [1968].
8 Y. Aharonov u. D. Bohm, Phys. Rev. 115, 485 [1959].

9 W. Heisenberg, Z. Naturforsch. 9 a, 292 [1954].

10 T. D. Lee, Phys. Rev. 95, 1329 [1954]; W. Heisenber G, Z. Phys. 144, 1 [1956]; Nucl. Phys. 4, 532 [1957]; 5, 195 [1958].

11 M. Karowski, Z. Naturforsch. 24 a, 510 [1969].

12 G. Källen u. W. Pauli, Kong. Dansk Vid. Selsk. Mat. Fys. Medd. 30, No. 7 [1955].

13 T. D. LeE u. G. C. WICK, Nucl. Phys. B 9, 209 [1969]; R. E. Cutкosky, P. V. Landshoff, D. I. Olive u. J. C. PolKinghorne, Nucl. Phys. B 12, 281 [1969] ; H. P. DürR u. E. Seiler, Nuovo Cim. 66 A, 734 [1970] ; A. M. Gleeson, R. J. Moore, H. Rechenberg u. E. C. G. Sudarshan, Phys. Rev. D 4, 4 [1971]

14 E. C. G. Sudarshan u. C. A. Nelson, Quantum Field Theories with Shadow States I, II. Preprint CPT 94, 95. Austin, Texas (March 1971).

15 M. Karowski, Private Mitteilung.

\title{
Relativistic, Three-Dimensional Equations for Low-Energy Two-Nucleon Systems
}

\author{
K. ERKelenZ and K. Holinde \\ Institut für Theoretische Kernphysik der Universität Bonn, Bonn, W.-Germany \\ (Z. Naturforsch. 28 a, 353--361 [1973] ; received 30 December 1972) \\ Dedicated to Professor KONRAD Bleuler on the occasion of his sixtieth birthday
}

\begin{abstract}
The manifestly covariant Bethe-Salpeter equation is reduced to relativistic, three-dimensional integral equations suitable for the dynamical treatment of the two-nucleon system at low energies. The reduction is achieved by restricting one of the nucleons to the mass shell. The resulting twonucleon scattering equations and bound state equations are Schrödinger-like field equations containing relativistic kinematical corrections. The transformation of these equations to the ordinary Schrödinger (or Lippmann-Schwinger) -equation is discussed. Intimately connected with the reduction is the derivation of a meson field mediated two-nucleon potential containing meson retardation effects and adequate for the application to the two-nucleon system and nuclear structure problems.
\end{abstract}

\section{Introduction}

The purpose of this paper is twofold. We wish to derive from first principles relativistic, three-dimensional off-shell equations for the dynamical treatment of the strongly interacting two-nucleon system at low energies *. The interacting field Hamiltonian appearing in these Schrödinger-like field equations should have the retarded nature of the meson field mediated interactions and must be appropriate for the application in nuclear physics problems.

In order to perform this program we start from the most orthodox, manifestly covariant approach to the relativistic two-body problem in quantum field theory, i. e. the four-dimensional (off-shell) Bethe-

Reprint requests to Dr. K. Erkelenz, Institut für Theoretische Kernphysik der Universität Bonn, D-5300 Bonn, Nußallee 16.
Salpeter (BS)-equation ${ }^{1,}$. In the low-energy region one expects that the intermediate state nucleons in the BS-equation remain close to their mass-shells. For spinless particles this expectation has been justified to some extent by Gross ${ }^{3}$ who showed that the graphs contributing to low-energy scattering can be well approximated by their positive-energy nucleon poles. Thus, in the low-energy region, it should be a good approximation if we calculate the BS-equation restricting one of the nucleons to its (positiveenergy) mass-shell. This requirement reduces the four-dimensional BS-equation to a three-dimensional off-shell equation. The dynamical equation so derived contains relativistic kinematical corrections and, as we want to stress, a meson field mediated inter-

\footnotetext{
* In a subsequent paper these equations will be used to analyse the low-energy region two-nucleon system.
} 
action which includes the meson retardation effects due to covariant perturbation theory.

The main motivation for using relativistic, threedimensional off-shell equations lies in the increasing number of nuclear structure problems which seem to require inclusion of relativistic effects like relativistic kinematical corrections and meson retardation ${ }^{4-8}$. The short-range repulsion of the twonucleon interaction generates strong high-momentum components in the two-nucleon wave function at all scattering energies. In nuclei the relativistic components of the nuclear wave functions are associated with such phenomena as relativistic kinematical corrections, nuclear short-range correlations, mesonic degrees of freedom and baryon resonances. It is very unlikely that such components are described correctly by a non-relativistic dynamical equation. Furthermore, as we have shown recently (see Refs. ${ }^{4,5}$ ), the meson retardation in the interaction strongly influences the values of meson coupling constants, the low-energy two-nucleon data and the nuclear matter data.

Intimately connected with our procedure of reducing the BS-equation is the derivation of a relativistic off-shell potential appropriate for the application in the two-nucleon system. The potential concept adopted here is that any interaction field Hamiltonian appearing in a three-dimensional dynamical equation may be referred to as a potential. In case this equation can be transformed into an ordinary Lippmann-Schwinger(LS)-equation the corresponding potential is adequate for application in the manybody Schrödinger equation. We stress that for reasons of consistency a potential applied in the manybody Schrödinger equation should have been unitarized also in a (two-body) Schrödinger (or LS) equation. That is because there does not exist a covariant many-body theory being the generalization to the multiparticle case of a covariant two-particle equation.

Our approach to the fully covariant BS-equation differs from the symmetric approaches proposed by Blancenbecler and Sugar ${ }^{9}$ (BBS) and ThompSON ${ }^{10}$ in the fact that we restrict one of the particles to its mass shell, henceforth cited as non-symmetric case. To our knowledge, a kinematical restriction of this type has been first proposed by Gross (see Ref. ${ }^{3}$ ) to derive covariant three-dimensional equations from straightforward perturbation theory. This method, however suffers from a rather arbitrary elimination of the negative energy states and is useful only in the static limit, i. e. the two nucleons are taken to be strictly fixed which implies that $(\mu / m)^{2} \ll 1$. $m$ and $\mu$ are the masses of the nucleon and the exchanged particles, respectively. In contrast to BBS and Thompson, in the non-symmetric case meson retardation effects in the meson field mediated potential are preserved in any order of the potential expansion, see Equation (3.15). The inclusion of these important effects even in the lowest order of the interaction kernel (compare Refs. ${ }^{4,5}$ ) may be an advantage of our approach.

In Section 2 we present the four-dimensional BSequation and discuss the field-theoretic concept underlying our definition of a two-nucleon potential. We mention that this concept is close to that of Charap, Fubini and Tausner ${ }^{11}$. In Section 3.1 we outline the arguments used to reduce the BS-equation and give the resulting relativistic, three-dimensional scattering equation. Section 3.2 contains the transformation of this equation into the ordinary LS-equation. In Section 3.3 we discuss the bound state (or homogeneous) counterpart of the scattering equation. For a detailed review of the different three-dimensional and unitary approaches to the BSequation we refer to Ref. ${ }^{12}$.

\section{Four-Dimensional BS-Equation and the Effective Interaction Kernel}

Bethe and Salpeter have shown on the basis of Feynman-graphical considerations that the fieldtheoretic amplitude for the two-nucleon scattering process can be described as the solution of the manifestly covariant, four-dimensional off-shell equation

$$
\begin{aligned}
& M\left(q^{\prime}, q \mid P\right)=K\left(q^{\prime}, q \mid P\right) \\
& \quad+\int \mathrm{d}^{4} k K\left(q^{\prime}, k \mid P\right) G(k, P) M(k, q \mid P) .
\end{aligned}
$$

This equation, of course, is to be regarded as an operator equation in the spin and isotopic spin space of the incoming and outgoing nucleons. The energymomentum conservinig variables are definend as in Fig. 1 where a symbolic representation of Eq. (2.1) is given. We use Eq. (2.1) in the center-of-mass frame, i. e. $P=\left(P_{0}, 0\right)$. Note that, in general, the particles have not to be restricted to their mass shells; that means, the time-like components of the relative momenta are unequal to zero. The interaction kernel $K\left(q^{\prime}, q \mid P\right)$ is the infinite sum of all 

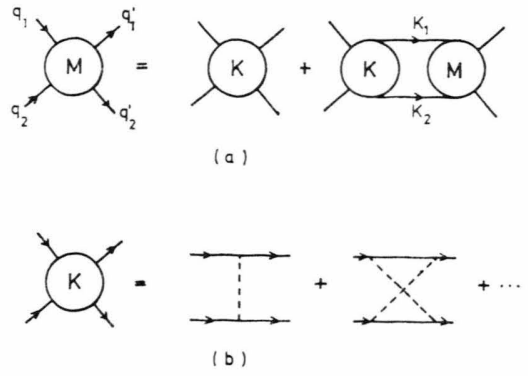

$$
\begin{array}{lll}
q=q_{1}-q_{2}: & k=k_{1}-k_{2} ; & q^{\prime}=q_{1}^{\prime}-q_{2}^{\prime} \\
p=q_{1}+q_{2}, & p=k_{1}+k_{2} & p=q_{1}^{\prime}+q_{2}^{\prime}
\end{array}
$$

Fig. 1. Graphic representation of the BS-equation (a) and the irreducible interaction kernel up to the fourth-order graph. The nucleons (solid lines) are not restricted to their mass shells.

possible irreducible two-particle diagrams $V^{(2 n) 13}$, i. e.

$$
K\left(q^{\prime}, q \mid P\right)=\sum_{n} V^{(2 n)}\left(q^{\prime}, q \mid P\right) .
$$

The superscript refers to successive orders in the coupling constant of the meson field mediated twonucleon graphs, see Figure 1. $G(k, P)$ is the relativistic, free two-nucleon Green's function,

$G(k, P)=$

$-\frac{i}{(2 \pi)^{4}} \frac{1}{\left[\frac{1}{2} P^{*}+k^{*}-m+i 0\right]_{1}\left[\frac{1}{2} P^{*}-k^{*}-m+i 0\right]_{2}}$

where the Feynman slash denotes the contraction of $a$ with the Dirac matrices.

The main justification for using the BS-equation is that it probably achieves better convergence than the straightforward perturbation expansion in the meson coupling constants. An exact solution, however, is evidently hopeless since, among other difficulties, the kernel cannot be given in closed form (for details see Ref. ${ }^{2}$ ). Thus we propose a three-dimensional approach to the BS-equation fairly easy to work with and designed to represent the BS-amplitude for low energy systems to a good approximation.

For that reason we consider the two-particle Green's function $G$. Its outstanding characteristic is that it can produce also contributions from inelastic states and from states with negative energies. Therefore, in the low-energy region of the two-nucleon system it seems to be reasonable to replace $G$ by a two-particle Green's function $g$ which can only pro-

\footnotetext{
* On the following pages the Feynman slash will be marked
} by an asterisk $a^{*}$ and also $P^{*}, k^{*}$. duce two-particle cuts in the physical energy region. To state it in other words, one expects that the intermediate nucleons remain close to their mass shell and thus the negative-energy contributions (from far off the mass shell) are quite small. As has been mentioned already in the introduction this assumption is supported by the work of Gross, see Reference ${ }^{3}$.

Following BBS we write

$$
G=g+[G-g] .
$$

It is now a simple task to show that the equation (written in symbolic operator notation)

$$
M=W+W g M
$$

where the effective interaction kernel $W$ has to be determined from

$$
W=K+K[G-g] W
$$

is entirely equivalent to the BS-equation (2.1). The method applied here to solve Eq. (2.6) is to expand it in a Neumann series, i. e.

$$
W=\sum W^{(2 n)}
$$

where

$$
W^{(2)}=V^{(2)}
$$

$$
W^{(4)}=V^{(4)}+V^{(2)}[G-g] V^{(2)} \quad \text { etc. }
$$

If $W$ is calculated up to the order $n$ from Eq. (2.7) the corresponding amplitude calculated from Eq. (2.5) will be exact to the same order and approach the higher ones. It is important to realize that the definition of $W^{(4)}$ because of $V^{(2)} G V^{(2)}$ already involves an integration over intermediate momenta and depends, therefore, on the off-shell continuation of $V^{(2)}$. Thus, in order to calculate $W$, a field-theoretical description based on a Lagrangian is inevitably required.

As an example we give the second order contribution to the interaction kernel $W$ assuming pseudoscalar coupling (and isospin 1)

$W_{\pi}^{(2)}\left(q^{\prime}, q\right)=V_{\pi}^{(2)}\left(q^{\prime}, q\right)=-g_{\pi}^{2} \tau_{1} \cdot \tau_{2} \frac{\gamma_{5}^{(1)} \gamma_{5}^{(2)}}{\left(q^{\prime}-q\right)^{2}-m_{\pi}^{2}}$

Here, $m_{\tau}$ and $g_{\tau}{ }^{2}$ denote the mass and coupling constant of the pion; $\tau$ the nucleon isospin operator and $\gamma_{5}$ the Dirac matrix. The scalar product is defined by $q^{2}=q_{0}^{2}-\boldsymbol{q}^{2}$. The fourth-order kernel $W^{(4)}$ then corresponds to the familiar fourth-order crossed and ladder pion exchange diagrams and to the iteration of the OPE-kernel, respectively. 
If we can find a suitable simple choice of $g$ such that it reduces Eq. (2.5) to a three-dimensional (offshell) equation and that the operator $[G-g]$ is sufficiently small for low-energy systems, Eqs. (2.5), (2.6) and (2.7) represent our derivation of the potential and the equation, namely $(2.5)$, in which it is to be inserted. It should be mentioned that in case a pion mediated interaction $W_{\pi}$ is considered the potential definition avoids double-counting of graphs. However, more careful investigations to this point are needed if we employ interactions based on the exchange of meson resonances, i. e. boson exchange potentials.

\section{Reduction of Four-Dimensional Bethe-Salpeter Equation}

\subsection{Relativistic, Three-dimensional Equation}

Our three-dimensional approach to the Bethe-Salpeter equation is based on the (kinematical) assumption that one particle (in its initial, intermediate and final state) is on its mass-shell (non-symmetric case ${ }^{14}$. If particle one is on the mass-shell then

$$
\left(\frac{1}{2} P+q\right)^{2}=\left(\frac{1}{2} P+k\right)^{2}=\left(\frac{1}{2} P+q^{\prime}\right)^{2}=m^{2}
$$

and hence, in the c. m. frame,

$q_{0}=E_{q}-\frac{1}{2} P_{0}, \quad k_{0}=E_{\mathrm{k}}-\frac{1}{2} P_{0}, q_{0}^{\prime}=E_{q^{\prime}}-\frac{1}{2} P_{0}$,

where $E_{p}{ }^{2}=\boldsymbol{p}^{2}+m^{2}$. Thus the amplitude $M\left(q^{\prime}, q \mid P_{0}\right)$ and interaction $W\left(q^{\prime}, q \mid P_{0}\right)$ no longer depend on the full four-momentum but only on the three-momentum and the parametric energy $P_{0}$.

Let us now introduce a two-particle Green's function $g(k, P)$ which has the following properties:

i) $g\left(k, P_{0}\right)$ is singular only when particle 1 (or 2 ) is on its mass shell. This guarantees, different to the symmetric approach, that the important meson retardation effects of the potential are preserved in any order of the potential expansion (2.7).

ii) The amplitude $M$, formally given by Eq. (2.5), satisfies the field theoretic condition of elastic unitarity (for simplicity the spin dependence is suppressed),

$$
i\left[M^{+}\left(\boldsymbol{q}^{\prime}, \boldsymbol{q} \mid P_{0}\right)-M\left(\boldsymbol{q}^{\prime}, \boldsymbol{q} \mid P_{0}\right)\right]=\frac{1}{(2 \pi)^{3}} \int \mathrm{d} \boldsymbol{k} \frac{m^{2}}{E_{k}{ }^{2}} \delta\left(2 E_{k}-P_{0}\right) M^{+}\left(\boldsymbol{q}^{\prime}, \boldsymbol{k} \mid P_{0}\right) M\left(\boldsymbol{k}, \boldsymbol{q} \mid P_{0}\right),
$$

where $P_{0}=2 E_{q}$. This ensures that in the physical energy region the two-particle cut structure of $g\left(k, P_{0}\right)$ is identical with that of the relativistic Green's function $G\left(k, P_{0}\right)$.

Thus, like for the symmetric choice of $g$ (see Ref. $\left.{ }^{9},{ }^{10}\right)$, the contributions of $V^{(2)} g V^{(2)}$ tend to cancel $V^{(2)} G V^{(2)}$ in the fourth-order potential $W^{(4)}$. This has been demonstrated for the BBS-choice of $g$ in Reference ${ }^{15}$. For the remaining crossed graph in $W^{(4)}, V^{(4)}$, we intuitively expect that it is smaller than the box graph because it requires two mesons to be present at the same time ${ }^{16}$. The cancellation based upon identical cut structure of $g$ and $G$ may also weaken higher-order contributions to $W$ which is desirable because of expansion (2.7).

The points i) and ii) will be acomplished by writing (in the c. m. frame)

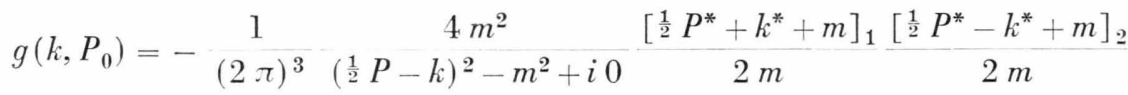

$$
\begin{aligned}
& \times \delta\left[\left(\frac{1}{2} P+k\right)^{2}-m^{2}\right] \Theta\left(\frac{1}{2} P_{0}+k_{0}\right)
\end{aligned}
$$

where $\Theta\left(q_{0}\right)$ is the conventional step function. Because of

$$
\delta\left[\left(\frac{1}{2} P+k\right)^{2}-m^{2}\right] \Theta\left(\frac{1}{2} P_{0}+k_{0}\right)=\frac{1}{2 E_{k}} \delta\left(\frac{1}{2} P_{0}+k_{0}-E_{k}\right)
$$

for the on-shell nucleon we obtain

$$
\left.\frac{\left[\frac{1}{2} P^{*}+k^{*}+m\right]_{1}}{2 m}\right|_{k_{0}=E_{k}-\frac{1}{2} P_{0}}=A_{1}^{+}(\boldsymbol{k})
$$

where $\mathrm{A}^{+}(\boldsymbol{k})$ is the positive-energy projector. For the off-shell nucleon we use the identity

$$
\frac{\left[\frac{1}{2} P^{*}-k+m\right]_{2}}{2 m}=\frac{E_{k}+\frac{1}{2} P_{0}-k_{0}}{2 E_{k}} \Lambda_{2}{ }^{+}(-\boldsymbol{k})+\frac{E_{k}-\frac{1}{2} P_{0}+k_{0}}{2 E_{k}}{A_{2}}^{-}(\boldsymbol{k})
$$


which, because of $k_{0}=E_{k}-\frac{1}{2} P_{0}$, specifies to

$$
\left.\frac{\left[\frac{1}{2} P^{*}-k^{*}+m\right]_{2}}{2 m}\right|_{k_{0}=E_{k}-\frac{1}{2} P_{0}}=\frac{P_{0}}{2 E_{k}}\left[A_{2}^{+}(-\boldsymbol{k})+\frac{2 E_{k}-P_{0}}{P_{0}} A_{2}^{-}(\boldsymbol{k})\right] .
$$

$A^{-}(\boldsymbol{k})$ is the negative-energy projector.

Collecting the results of Eqs. (3.5) and (3.6) the Green's function (3.4) reads as ${ }^{17}$

$$
g\left(k, P_{0}\right)=\frac{1}{(2 \pi)^{3}} m^{2} E_{k}^{2} \frac{\Lambda_{1}{ }^{+}(\boldsymbol{k})}{2 E_{k}-P_{0}-i c}\left[\Lambda_{2}^{+}(-\boldsymbol{k})+\frac{2 E_{k}-P_{0}}{P_{0}} \Lambda_{2}^{-}(\boldsymbol{k})\right] \delta\left(\frac{1}{2} P_{0}+k_{0}-E_{k}\right) .
$$

Inserting (3.7) into Eq. (2.5) we obtain the relativistic three-dimensional equation

$$
\begin{aligned}
& M\left(\boldsymbol{q}^{\prime}, \boldsymbol{q} \mid P_{0}\right)=W\left(\boldsymbol{q}^{\prime}, \boldsymbol{q} \mid P_{0}\right)+\frac{1}{(2 \pi)^{3}} \int \mathrm{d} \boldsymbol{k} \frac{m^{2}}{E_{k}{ }^{2}} W\left(\boldsymbol{q}^{\prime}, \boldsymbol{k} \mid P_{0}\right) A_{1}{ }^{+}(\boldsymbol{k}) \\
& 2 E_{k}-P_{0}-i o \\
& \cdot\left[A_{2}{ }^{+}(-\boldsymbol{k})+\frac{2 E_{k}-P_{0}}{P_{0}} A_{2}{ }^{-}(\boldsymbol{k})\right] M\left(\boldsymbol{k}, \boldsymbol{q} \mid P_{0}\right) .
\end{aligned}
$$

Here, the parametric energy $P_{0}$ may be taken as $P_{0}=2 E_{q}$ but then $q^{\prime}, k$ and $q$ must be taken as

$$
q^{\prime}=\left(\boldsymbol{q}^{\prime}, E_{q^{\prime}}-E_{q}\right), \quad k=\left(\boldsymbol{k}, E_{k}-E_{q}\right) \quad \text { and } \quad q=(\boldsymbol{q}, 0) .
$$

That means the three-dimensional potential $W$ is defined by $W\left(\boldsymbol{q}^{\prime}, \boldsymbol{q} \mid P_{0}\right)=\left.W\left(q^{\prime}, q \mid P_{0}\right)\right|_{q_{0}=0, q_{0}^{\prime}=E_{q^{\prime}}-E_{q}}$ and thus contains meson retardation effects in any order of the expansion (2.7) or, stated in other words, is continued off-energy shell according to the covariant perturbation theory. Consequently, in calculating the three-dimensional amplitude $M\left(\boldsymbol{q}^{\prime}, \boldsymbol{q} \mid P_{0}\right)$ via Eq. (3.8) off-shell effects are included even in case the OBE-model for the potential $W$, i. e. $W=V^{(2)}$, is applied. This is different from the three-dimensional symmetric approaches of BBS and Thompson.

In order to analyse the two-nucleon system we have to solve Equation (3.8). For that reason we observe that

and

$$
\begin{aligned}
& \Lambda^{+}(\boldsymbol{k})=\sum_{\lambda}\left|u_{\lambda}(\boldsymbol{k})\right\rangle\left\langle\bar{u}_{\lambda}(\boldsymbol{k})\right| \\
& \Lambda^{-}(\boldsymbol{k})=\sum_{\lambda}\left|v_{\lambda}(\boldsymbol{k})\right\rangle\left\langle\bar{v}_{\lambda}(\boldsymbol{k})\right|
\end{aligned}
$$

where $u(\boldsymbol{k})$ and $v(\boldsymbol{k})$ denote the positive- and negative-energy spinor of momentum $\boldsymbol{k}$ and helicity $\lambda$; $\bar{u}$ and $\bar{v}$ are their adjoints. This indicates that we have to introduce the following matrix elements of $M$,

$$
\mathfrak{M}^{++}\left(\boldsymbol{q}^{\prime}, \boldsymbol{q} \mid P_{0}\right)=\left\langle\bar{u}_{\lambda_{1}}\left(\boldsymbol{q}^{\prime}\right) \bar{u}_{\lambda_{2}^{\prime}}\left(-\boldsymbol{q}^{\prime}\right)|\boldsymbol{M}| \boldsymbol{u}_{\lambda_{1}}(\boldsymbol{q}) u_{\lambda_{2}}(-\boldsymbol{q})\right\rangle
$$

and

$$
\mathfrak{M}^{-+}\left(\boldsymbol{q}^{\prime}, \boldsymbol{q} \mid P_{0}\right)=\left\langle\bar{u}_{\lambda_{1}^{\prime}}\left(\boldsymbol{q}^{\prime}\right) \bar{v}_{\lambda_{2}^{\prime}}\left(\boldsymbol{q}^{\prime}\right)|M| u_{\lambda_{1}}(\boldsymbol{q}) u_{\lambda_{2}}(-\boldsymbol{q})\right\rangle \text {. }
$$

Furthermore, we have to consider the potential matrix elements

$$
\begin{aligned}
& \mathfrak{W}^{++}\left(\boldsymbol{q}^{\prime}, \boldsymbol{q} \mid P_{0}\right)=\left\langle\bar{u}_{\lambda_{1}^{\prime}}\left(\boldsymbol{q}^{\prime}\right) \bar{u}_{\lambda_{2}^{\prime}}\left(-\boldsymbol{q}^{\prime}\right)|W| u_{\lambda_{1}}(\boldsymbol{q}) u_{\lambda_{2}}(-\boldsymbol{q})\right\rangle, \\
& \mathfrak{W}^{+-}\left(\boldsymbol{q}^{\prime}, \boldsymbol{q} \mid P_{0}\right)=\left\langle\bar{u}_{\lambda_{1}^{\prime}}\left(\boldsymbol{q}^{\prime}\right) \bar{u}_{\lambda_{2}^{\prime}}\left(-\boldsymbol{q}^{\prime}\right)|W| u_{\lambda_{1}}(\boldsymbol{q}) v_{\lambda_{2}}(\boldsymbol{q})\right\rangle, \\
& \mathfrak{W}^{-+}\left(\boldsymbol{q}^{\prime}, \boldsymbol{q} \mid P_{0}\right)=\left\langle\bar{u}_{\lambda_{1}^{\prime}}\left(\boldsymbol{q}^{\prime}\right) \bar{v}_{\lambda_{2}^{\prime}}\left(\boldsymbol{q}^{\prime}\right)|W| u_{\lambda_{1}}(\boldsymbol{q}) u_{\lambda_{2}}(-\boldsymbol{q})\right\rangle
\end{aligned}
$$

and

$$
\mathfrak{W}^{--}\left(\boldsymbol{q}^{\prime}, \boldsymbol{q} \mid P_{0}\right)=\left\langle\bar{u}_{\lambda_{1}}\left(\boldsymbol{q}^{\prime}\right) \bar{v}_{\lambda_{2}^{\prime}}\left(\boldsymbol{q}^{\prime}\right)|W| u_{\lambda_{1}}(\boldsymbol{q}) v_{\lambda_{2}}(\boldsymbol{q})\right\rangle \text {. }
$$

Now, it is a straightforward task to show that the large-large components of $\mathfrak{M}$, i. e. $\mathfrak{M C}^{++}$, and the largesmall component $\mathfrak{M}^{-+}$are determined by a system of two coupled integral equations which reads as ${ }^{18}$

$$
\begin{aligned}
& \mathfrak{M}^{++}\left(\boldsymbol{q}^{\prime}, \boldsymbol{q} \mid P_{0}\right)=\mathfrak{W}^{++}\left(\boldsymbol{q}^{\prime}, \boldsymbol{q} \mid P_{0}\right)+\frac{1}{(2 \pi)^{3}} \int \mathrm{d} \boldsymbol{k} \frac{m^{2}}{E_{k}{ }^{2}} \frac{1}{2 E_{k}-P_{0}-i o} \\
& \times\left[\mathfrak{W}^{++}\left(\boldsymbol{q}^{\prime}, \boldsymbol{k} \mid P_{\mathbf{0}}\right) \mathfrak{M}^{++}\left(\boldsymbol{k}, \boldsymbol{q} \mid P_{\mathbf{0}}\right)+\frac{2 E_{k}-P_{0}}{P_{0}} \mathfrak{W}^{+-}\left(\boldsymbol{q}^{\prime}, \boldsymbol{k} \mid P_{0}\right) \mathfrak{M}^{-+}\left(\boldsymbol{k}, \boldsymbol{q} \mid P_{0}\right)\right] \\
& \mathfrak{M}^{-+}\left(\boldsymbol{q}^{\prime}, \boldsymbol{q} \mid P_{0}\right)=\mathfrak{W}^{-+}\left(\boldsymbol{q}^{\prime}, \boldsymbol{q} \mid P_{0}\right)+\frac{1}{(2 \pi)^{3}} \int \mathrm{d} \boldsymbol{k} \frac{m^{2}}{E_{k}{ }^{2}} 2 \frac{1}{2 E_{k}-P_{0}-i o} \\
& \times\left[\mathfrak{W}^{-+}\left(\boldsymbol{q}^{\prime}, \boldsymbol{k} \mid P_{\mathbf{0}}\right) \mathfrak{M}^{++}\left(\boldsymbol{k}, \boldsymbol{q} \mid P_{0}\right)+\frac{2 E_{k}-P_{0}}{P_{0}} \mathfrak{W}^{--}\left(\boldsymbol{q}^{\prime}, \boldsymbol{k} \mid P_{0}\right) \mathfrak{M}^{-+}\left(\boldsymbol{k}, \boldsymbol{q} \mid P_{0}\right)\right] .
\end{aligned}
$$


We mention that the appearance of $\mathfrak{M}^{-+}$in the calculation of the $\mathrm{NN}$-scattering is due to the fact that one particle is off its mass shell.

In order to define a potentil comparable with others and suitable for the application in the many-body Schrödinger equation we drop the term connected with $\mathfrak{S}^{--}$in Equation (3.12). This may be motivated to a certain extent in the following way. For low intermediate state momenta the kinematical factor in front of $\mathfrak{W}^{--}$is of the order $(m-m) / m$. Thus the contribution of $\mathfrak{W}^{--}$is strongly suppressed for low momenta. For large momenta the kinematical factor increases linearly with $\boldsymbol{k}$ but again its product with $\mathfrak{W S}^{--}$remains small since the asymptotic behaviour of $\mathfrak{W}^{--}$must be regularized by means of cut-offs or form factors which create a rapid fall-off of $\mathfrak{W G}^{--}$asymptotically; see the end of this section. Ultimately, of course, this approximation must be checked by integrating the correct equation system (3.11) and (3.12) numerically. This is under consideration using the helicity state formalism as extended by us to off-energy shell amplitudes ${ }^{19}$.

Omitting the $\mathfrak{W}^{--}$-term in Eq. (3.12) decouples the system and results in the equation

$$
\mathfrak{M}^{++}\left(\boldsymbol{q}^{\prime}, \boldsymbol{q} \mid P_{0}\right)=\mathfrak{W}_{\mathrm{eff}}\left(\boldsymbol{q}^{\prime}, \boldsymbol{q} \mid P_{0}\right)+\frac{1}{(2 \pi)^{3}} \int \mathrm{d} \boldsymbol{k} \frac{m^{2}}{E_{k}{ }^{2}} \mathfrak{W}_{\mathrm{eff}}\left(\boldsymbol{q}^{\prime}, \boldsymbol{k} \mid P_{0}\right) \frac{1}{2 E_{k}-P_{0}-i o} \mathfrak{N}^{++}\left(\boldsymbol{k}, \boldsymbol{q} \mid P_{0}\right)
$$

where $\quad \mathfrak{W}_{\mathrm{eff}}\left(\boldsymbol{q}^{\prime}, \boldsymbol{q} \mid P_{0}\right)=\mathfrak{W}^{++}\left(\boldsymbol{q}^{\prime}, \boldsymbol{q} \mid P_{0}\right)+\frac{1}{(2 \pi)^{3}} \int \mathrm{d} \boldsymbol{k} \frac{m^{2}}{E_{k}{ }^{2}} \mathfrak{W}^{+-}\left(\boldsymbol{q}^{\prime}, \boldsymbol{k} \mid P_{0}\right) \mathfrak{W}^{-+}\left(\boldsymbol{q}, \boldsymbol{k} \mid P_{0}\right)$.

$\mathfrak{W}_{\text {eff }}$, occuring in the Schrödinger-like equation (3.13), may be considered as an effective potential. Its outstanding property is that it provides a model independent explanation of the repulsive core in the two-nucleon interaction directly based on the Lorentz invariance of our approach. The reason for that is that the second term on the right hand side of Eq. (3.14) dominates at short distances and falls off more rapidly than $\mathfrak{W}^{++}$at large distances as has been shown by Gross ${ }^{20,} 21$ in the scheme of the OBE-model.

We terminate this section with a general discussion about the potential concept of the two-nucleon interaction which, as we believe, may be suitable to provide a "zero-parameter" description of the lowenergy two-nucleon system. The relationship between the mass of the exchanged mesons and the range of the interaction for Yukawa-type coupling indicates that the classical region $(r \gtrsim 1.5 \mathrm{fm})$ of the potential is dominated by the OPE-contribution to $W$. In our approach this reads as

$$
\begin{aligned}
& V_{\pi}^{(2)}\left(\boldsymbol{q}^{\prime}, \boldsymbol{q}\right) \gamma_{5}^{(1)} \gamma_{5}^{(2)} \\
&=-g_{\pi}^{2} \tau_{1} \cdot \tau_{2}(3.15) \\
&\left(E_{q^{\prime}}-E_{q}\right)^{2}-\left(\boldsymbol{q}^{\prime}-\boldsymbol{q}\right)^{2}-m_{\pi}^{2}
\end{aligned}
$$

which means that the meson retardation effects are included. Since the integral Eq. (3.14) is not of Fredholm type for the OPE-contribution the latter must be modified with a convergence generating factor, generally called form factor, which can be attributed to the finite size of the nucleons. For that rea- son one considers a particular class of Feynman diagrams wtih vertex corrections which contribute to the irreducible kernel $(2.2)^{22,23}$. In the eikonal approximation, as has been shown by WoLOSHYN and $\mathrm{JACKSON}^{23}$, this infinite class can be summed and yields an analytic expression. The result is that the coupling constant $g_{\pi}$ must be replaced by $g_{\pi} F\left(\left(q^{\prime}-q\right)^{2}\right)$ where $F$ denotes a form factor exponentially decreasing with the momentum transfer. We believe that this approach is less ambiguous than the introduction of purely phenomenological form factors especially because the parameters can be linked with those of other processes possible to be described with the eikonal approach but independent of the nucleon-nucleon system, as the magnetic form factor of the proton, see Reference ${ }^{23}$.

The dynamical region $(0.5 \lesssim r \lesssim 1.5)$ is aside from meson- and baryon resonances which contribute to $W^{(2)}$ (as the $\varrho$ - and $\omega$-vector meson exchange) and to $W^{(4)}$ (as the $N_{33}^{*}$ and $N_{11}^{*}$ resonances), affected by the TPE-exchange terms included in $W^{(4)}$. The contributions of uncorrelated higher multimeson exchanges ${ }^{24}$ are expected to be unimportant. Some motivation for this expectation is given in Reference ${ }^{15}$. Naturally, because of the strong interaction between pions a complete analysis of the dynamical region should also take into account the correlated $2 \pi$-exchange in the S-wave state. This, for instance, can be done in the way proposed by Снемтов, DURSO and RiskA ${ }^{25}$. 
In the core region $(r \lessgtr 0.5)$ a multitude of complex phenomena contribute to the potential. Fortunately, the repulsive core of the potential keeps the two nucleons well apart so that the majority of the short range contributions included in $W$ are masked by the form factors necessary to renormalize all the forementioned diagrams. In the scheme of the OBEmodel the repulsive core certainly is a combined effect of the vector meson exchange contributions and the off-diagonal terms in (3.14).

\subsection{Transformation to Ordinary Lippmann- Schwinger Equation}

In order to define a nuclear potential adequate for nuclear structure calculations (in the sense we have discussed in the introduction) Eq. (3.13) must be reduced to the ordinary LS-equation. This, however, cannot be done in a straightforward way but only in a certain limit because the causal part of the Green's function in Eq. (3.13) is not of Schrödin- ger type. In order to avoid the adiabatic limit, which would be rather doubtful since intermediate state momenta are involved, we propose a different way of reduction.

Calculating an off-energy shell $q$-space OBEP (see Ref. ${ }^{4}$ ) which describes the empirical nuclear phase shifts accurately up to $350 \mathrm{MeV}$ (lab. energy), we have seen that it peaks around three-momenta of about $1 \mathrm{GeV} / \mathrm{c}$ and falls off rapidly with increasing momenta because of the convergence generating form factors. There are strong indications that this will be a general feature of any meson field mediated $q$-space potential designed to fit the low-energy scattering data. Thus we assume that such a potential (3.14) is peaked in the range of momenta $\boldsymbol{k}$ with

$$
\left|2 \sqrt{\boldsymbol{k}^{2}+m^{2}-P_{0}}\right| / P_{0} \ll 1
$$

and converges faster than $|\boldsymbol{k}|^{-1}$ outside this region. This suggests, observing that

$$
\frac{1}{2 E_{k}-P_{0}-i o}=\frac{\frac{1}{2} P_{0}}{E_{k}^{2}-\frac{1}{4} P_{0}^{2}-i o} \cdot\left(1+\frac{1}{2} \frac{2 E_{k}-P_{0}}{P_{0}}\right),
$$

that the dynamical Eq. (3.13) can be approximated by

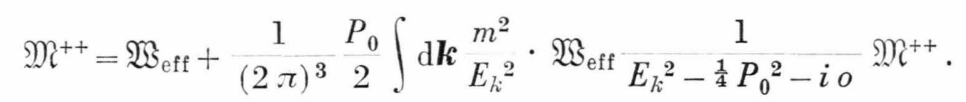

We stress the point that the assumption made to derive Eq. (3.18) from (3.13) only influences the analytic expression of the Green's function $g$ but without changing its cut structure. Thus the desirable feature of $g$ as discussed under point ii) in Sect. 3.1 remains unaltered. Keep in mind that the aim of our three-dimensional approach to the BS-equation is to determine an (off-shell) $g$ in such a way that the operator $[G-g]$ is small in the low-energy region rather than to provide an utmost exact $g$.

In order to have a first check of Eq. (3.18) we have compared the nuclear phase shifts of this equation with those of Eq. (3.13) using our recent $q$ space OBEP ${ }^{26}$, see Reference ${ }^{4}$. (In terms of the present effective potential this corresponds to $\mathfrak{W}_{\text {eff }}$ $=\mathfrak{W}^{(2)}{ }^{27}$, i. e. off-diagonal and higher order contributions have been ignored.) In the energy region up to $350 \mathrm{MeV}$ the S-wave phases (see Fig. 2) differ less than $3 \%$ in the average 27 while the higher partial wave phases are in excellent agreement. This result may be considered as a first justification of

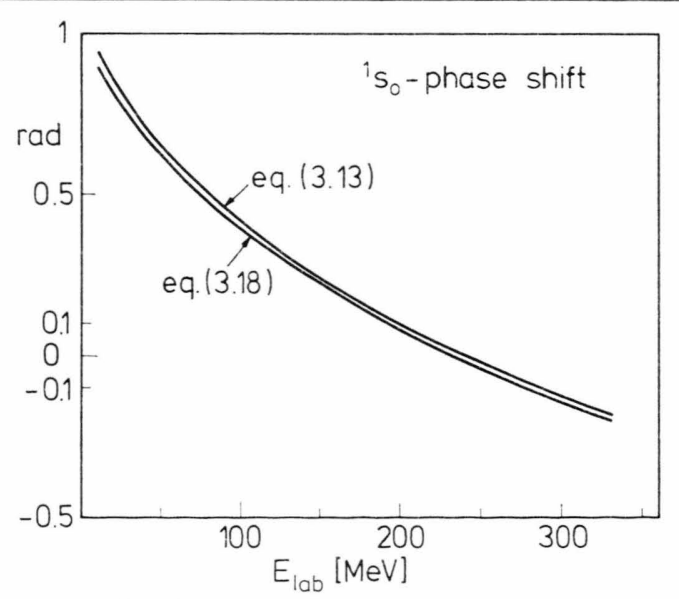

Fig. 2. The ${ }^{1} \mathrm{~S}_{0}$-phase shifts as function of laboratory energy calculated with the dynamical equations (3.13) and (3.18) for our $q$-space OBEP $\underset{\mathfrak{M}^{(2)++}}{\operatorname{Ref}}{ }^{4}$ ). This corresponds to $\mathfrak{W}_{\mathrm{eff}}=$

Eq. (3.18) (which, of course, needs further investigation) and, at the same time, as motivation for the omission of the $\mathfrak{S}^{--}$-term in Eq. (3.12) ; compare the discussion in Section 3.1. 
In order to show that Eq. (3.18) is completely equivalent to the ordinary LS-equation in the sense that both equations produce identical phase shifts we have to introduce the (uniquely determined) quantities

$$
\mathrm{t}^{++}\left(\boldsymbol{q}^{\prime}, \boldsymbol{q} \mid P_{0}\right)=\frac{P_{0}}{2} \frac{V m}{E_{q^{\prime}}} \Re_{i^{++}}\left(\boldsymbol{q}^{\prime}, \boldsymbol{q} \mid P_{0}\right) \frac{V m}{E_{q}}
$$

and

$$
\mathfrak{B}_{\mathrm{eff}}\left(\boldsymbol{q}^{\prime}, \boldsymbol{q} \mid P_{0}\right)=\frac{P_{0}}{2} \frac{V m}{E_{q^{\prime}}} \mathfrak{W}_{\mathrm{eff}}\left(\boldsymbol{q}^{\prime}, \boldsymbol{q} \mid P_{0}\right) \frac{V m}{E_{q}} .
$$

The application of (3.19) and (3.20) in (3.18) results in the ordinary LS-equation,

$$
\mathfrak{t}^{++}=\mathfrak{B}_{\text {eff }}+\frac{1}{(2 \pi)^{3}} \mathfrak{B}_{\text {eff }} \boldsymbol{k}^{2}-\boldsymbol{q}^{2}-i o{ }^{\mathrm{t}^{++}} .
$$

Thus, in the sense of our nuclear potential concept, the effective potential $\mathfrak{W}_{\text {eff }}$ unitarized by Eq. (3.18) and modified according to relation (3.20), that is $\mathfrak{Z}_{\text {eff }}$, should be adequate for application in the many-body Schrödinger equation. We stress the point that the relativistic kinematical corrections of the nucleon motion linked with the Green's function (3.18) are now included in $\mathfrak{B}_{\text {eff }}$.

\subsection{Three-dimensional Bound State Equations}

The bound state (or homogeneous) counterpart of the two-nucleon scattering BS-equation may be written as (see Ref. ${ }^{2}$ )

$\psi(q, P)=\int \mathrm{d}^{4} k K(q, k \mid P) G(k, P) \psi(k, P)$

where the amplitude $\psi(q, P)$ is a 16-component spinor, $K(q, k \mid P)$ the irreducible kernel $(2.2)$ and $G(k, P)$ the relativistic Green's function (2.3). A
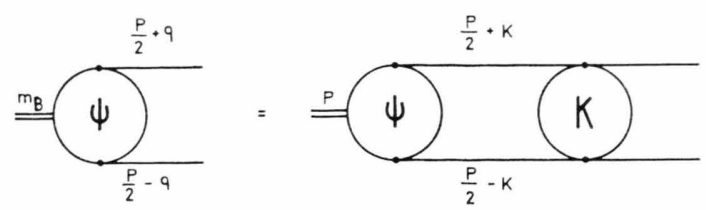

Fig. 3. The bound state BS-equation $(3.22) \cdot m_{B}$ is the bound state mass.

graphical representation of Eq. (3.22) is given in Figure 3.

In order to make the bound state BS-equation tractable we proceed as in the scattering case. That means, we rewrite Eq. (3.22) in the form (using operator notation)

$$
\psi=W g \psi
$$

where the interaction kernel $W$ satisfies Equation (2.6). It needs simple operator algebra to show the equivalence of Eqs. (3.22) and (3.23). As in the scattering case we shall consider Eq. (3.23) in the momentum space to take advantage of the fact that in the $q$-space all the causal functions included in (3.23) have a simple structure.

The Green's function (3.7) is employed to reduce (3.23) to a three-dimensional (relativistic) bound state equation. The result is, in the rest frame of the bound state, a system of two coupled integral equations similar to that for the scattering case [Eqs. (3.11) and (3.12)]. The difference is that the inhomogeneous terms have to be omitted, $P_{0}$ now represents the bound state mass $m_{\mathrm{B}}{ }^{28}$, and the amplitudes $\mathfrak{M}^{++}, \mathfrak{M}^{-+}$have to be replaced by the large-large and large-small components of $\psi$, i. e. $\psi^{++}$and $\psi^{-+}$. Naturally, these components also depend on the helicities of the two bound state nucleons.

Following now the line of argumentation used to derive the scattering Eq. (3.13) from the system (3.11), (3.12) we obtain the bound state counterpart of Eq. (3.13) which reads as

$$
\psi^{++}\left(\boldsymbol{q}, P_{0}\right)=\frac{1}{(2 \pi)^{3}} \int \mathrm{d} \boldsymbol{k} \frac{m^{2}}{E_{k}^{2}} \mathfrak{W}_{\text {eff }}\left(\boldsymbol{q}, \boldsymbol{k} \mid P_{0}\right) \underset{2 E_{k}-P_{0}}{1} \psi^{++}\left(\boldsymbol{k}, P_{0}\right)
$$

where the effective potential $\mathfrak{W}_{\text {eff }}\left(\boldsymbol{q}, \boldsymbol{k} \mid P_{0}\right)$ is given by Eq. (3.14) with $P_{0}=m_{\mathrm{B}}$. Thus Eq. (3.24) allows to include negative energy contributions to the bound state wave function $\psi^{++}$. It has been pointed out by Remler (see Ref. ${ }^{6}$ ), who proposed a practical theory of the relativistic deuteron wave function, that such contributions may influence the short range part of the deuteron waves to an appreciable extent ${ }^{29}$.

In the following we focuse our attention to the bound state counterpart of Equation (3.18). It is

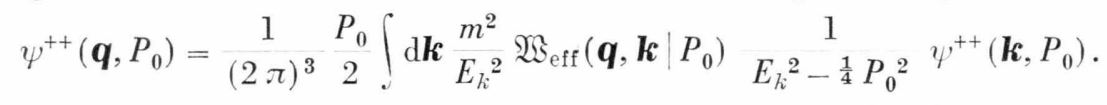

The underlying assumption in deriving Eq. (3.25) from (3.24) again is that the potential $\mathfrak{W}_{\text {eff }}$ designed to fit the two-nucleon low-energy data falls off rapidly for momenta large compared with $1 \mathrm{GeV} / \mathrm{c}$. The validity of this assumption for the bound state case is under investigation. 
The advantage of Eq. (3.25) is that it permits a direct transformation to the ordinary bound state Schrödinger equation. Using in (3.25) the transformation

$\varphi^{++}\left(\boldsymbol{q}, P_{0}\right)=\frac{1}{E_{q}{ }^{2}-\frac{1}{4} P_{0}{ }^{2}} \frac{\sqrt{m}}{E_{q}} \psi^{++}\left(\boldsymbol{q}, P_{0}\right)$

and Eq. (3.20) with $P_{0}=m_{\mathrm{B}}$, we obtain

$$
\begin{aligned}
\varphi^{++}\left(\boldsymbol{q}, P_{0}\right)= & \frac{m}{E_{q}{ }^{2}-\frac{1}{4} P_{0}{ }^{2}} \frac{1}{(2 \pi)^{3}} \int \mathrm{d} \boldsymbol{k} \\
& \cdot \mathfrak{N}_{\mathrm{eff}}\left(\boldsymbol{q}, \boldsymbol{k} \mid P_{0}\right) \varphi^{++}\left(\boldsymbol{k}, P_{0}\right) .
\end{aligned}
$$

For calculational reasons this equation can be simplified making the weak binding assumption for the two-nucleon bound state, that means we use $P_{0} \cong 2 \mathrm{~m}$ or $P_{0}=2 m-B$ with $B \ll 2 m$. For the deuteron $(B \cong 2 \mathrm{MeV})$ this is extremely accurate. Thus the kinematical factor in (3.27) can be replaced by

$$
E_{q}^{2}-\frac{1}{4} P_{0}^{2} \cong \boldsymbol{q}^{2}+B
$$

which reduces Eq. (3.27) formally to the ordinary bound state Schrödinger equation. Note that now $\mathfrak{B}_{\text {eff }}=\mathfrak{B}_{\text {eff }}\left(\boldsymbol{q}, \boldsymbol{k} \mid P_{0}\right)$ with $P_{0}=2 \mathrm{~m}$. The corresponding wave function is normalized according to $\left\langle\varphi^{++} \mid \varphi^{++}\right\rangle=1$. We note that no Wick rotation

1 E. Salpeter and H. Bethe, Phys. Rev. 84, 1232 [1951].

2 N. Nakanishi, Progr. of Theor. Phys. Suppl. No. 43 [1969] ; Extensive Review of the Theory of the BS-equation.

3 F. Gross, Phys. Rev. 186, 1448 [1969].

${ }^{4}$ K. Holinde, K. Erkelenz, and R. Alzetta, Nucl. Phys. A 194, 161 [1972].

5 K. Holinde, K. Erkelenz, and R. Alzetta, Nucl. Phys. A 198, 598 [1972].

${ }^{6}$ E. Remler, The Relativistic Deuteron Wave Function, preprint 1971, Williamsburg, Virginia.

7 G. E. Brown, A. Jackson, and T. Kuo, Nucl. Phys. A 133, 481 [1969].

8 M. Fortes and A. Jackson, Nucl. Phys. A 175, 449 [1971] ; Phys. Lett. 38 B, 277 [1972].

9 R. Blancenbecler and R. Sugar, Phys. Rev. 142, 1051 [1966].

10 R. Thompson, Phys. Rev. D 1, 110 [1970].

11 J. Charap and S. Fubini, Nuov. Cim. XIV, 540 [1959]; J. Charap and M. Tausner, Nuov. Cim. XVIII, 316 [1960].

12 K. Erkelenz and K. Holinde, Relativistic OBEP. TwoNucleon and Nuclear Matter Data, to be published in Phys. Reports.

13 Such diagrams cannot be separated into disjoint pieces by cutting nucleon lines only.

14 The non-symmetric treatment of the two particles may be considered to be an disadvantage of our approach. On the other hand, its must also be kept in mind that proposing a soluble equation reduced from the BS-equation necessarily implies to accept defects which, however, may be small in the domain of validity the approach is designed for.

15 M. Partovi and E. Lomon, Phys. Rev. D 2, 1999 [1970].

16 For the scalar scalar-exchange model this has been proved by F. Gross (Ref. ${ }^{3}$ ). (transformation from Minkowski to Euclidean metric) was necessary to derive the Schrödinger equation.

The partial wave decomposition of Eq. (3.27) (and the others mentioned above) using the helicity state representation can be found in Reference ${ }^{30}$. There we also give the experimental bound state quantities as the quadrupole and magnetic moment of the deuteron in terms of the helicity state basis.

The scattering and bound state equations discussed here can be applied also to the nucleon-antinucleon system. In this case the potential contributions due to meson exchange with positive $G$-parity remain unchanged while those mediated by mesons with negative $G$-parity (as the $\pi$ - and $\omega$-meson) change sign.

It is a pleasure to acknowledge useful conversations with Professor Konrad Bleuler. One of us (K.E.) would like to thank Professor G. E. BRown for enlightening comments and the Nordita, Copenhagen, for hospitality. We are grateful to Drs. A. JACKSON and R. Woloshyn, Nordita, for considerable provocation which induced us to revise the representation of the present paper. We wish to thank Dr. L. LundBerG, Nordita, for showing us the paper of C. FRONSDAL and R. HUFF ${ }^{31}$ which has some ideas in common with ours.

${ }^{17}$ In the spinless case the corresponding Green's function is

$$
g\left(k, P_{0}\right)=\frac{1}{(2 \pi)^{3}} \frac{1}{2 P_{0} E_{k}} \frac{\delta\left(P_{0} / 2+k_{0}-E_{k}\right)}{2 E_{k}-P_{0}-i o} .
$$

18 Here we have suppressed the helicity state indices and their summation in the intermediate states.

19 K. Erkelenz, R. Alzetta, and K. Holinde, Nucl. Phys. A 176, 413 [1971].

20 F. Gross, A New Theory of Nuclear Forces, preprint SLAC-PUB-1138 (1972).

21 We would like to thank Prof. G. E. BRown who brought to our attention the work of F. Gross. This paper has many ideas in common with ours. As a main result of this paper we mention that the one pion exchange dominates the repulsive core which can be considered as a new role of the pion in the two-nucleon interaction.

22 M. Islam, Nuov. Cim. 5 A, 315 [1971].

${ }^{23}$ R. Woloshyn and A. Jackson, Nucl. Phys. A 185, 131 [1972].

24 That means those not resonating in a single boson.

25 M. Chemtob, J. Durso, and D. Riska, Nucl. Phys. B 38, 141 [1972] ; see also W. Cottingham et al., $\mathrm{N}-\mathrm{N}$ interaction from $\pi-\mathrm{N}$ phase shift analysis, preprint IPNO TH $72-23$, Paris.

26 Without the minimal relativity factor.

27 This, for instance, corresponds to a variation of the isoscalar scalar coupling constant $g_{\sigma_{0}}^{2}$ of less than $1 \%$.

28 The bound state pole $s=P_{0}^{2}=m_{B}^{2}$ is related to the binding energy (or mass defect) $B(\geqq 0)$ by $-\mathrm{B}=P_{0}-2 \mathrm{~m}$.

29 Recently, F. GRoss has confirmed this conception, see Reference ${ }^{20}$.

30 R. Alzetta, K. Erkelenz, and K. Holinde, Nucl. Phys. A 185, 459 [1972].

31 C. Fronsdal and R. Huff, Phys. Rev. D 3, 933 [1971]. 